\title{
An evolutionary model of price competition among spatially distributed firms
}

\author{
Ludo Waltman ${ }^{1,2}$, Nees Jan van Eck ${ }^{1,2}$, Rommert Dekker ${ }^{2}$, and Uzay Kaymak ${ }^{2}$ \\ ${ }^{1}$ Centre for Science and Technology Studies, Leiden University, The Netherlands \\ \{waltmanlr, ecknjpvan\}@cwts.leidenuniv.nl \\ ${ }^{2}$ Econometric Institute, Erasmus School of Economics, Erasmus University Rotterdam, \\ The Netherlands \\ \{rdekker,kaymak\}@ese.eur.nl
}

EI 2011-09

\begin{abstract}
Various studies have shown the emergence of cooperative behavior in evolutionary models with spatially distributed agents. We investigate to what extent these findings generalize to evolutionary models of price competition among spatially distributed firms. We consider both one- and twodimensional models, and we vary the amount of information firms have about competitors in their neighborhood. Our computer simulations show that the emergence of cooperative behavior depends strongly on the amount of information available to firms. Firms tend to behave most cooperatively if they have only a very limited amount of information about their competitors. We provide an intuitive explanation for this phenomenon. Our simulations further indicate that three other factors in our models, namely the accuracy of firms' information, the probability of experimentation, and the spatial distribution of consumers, have little effect on the emergence of cooperative behavior.
\end{abstract}

\section{Introduction}

The phenomenon of cooperative behavior among individuals in social, economic, and biological systems has been fascinating researchers already for a long time. An important topic in the economic and biological literature is the emergence of cooperative behavior among individuals who are pursuing their self-interest. Researchers aim to identify the conditions under which the emergence of cooperative behavior among such individuals is possible.

In an economic context, the best-known explanation of cooperative behavior is probably the one based on the idea of reciprocity in repeated encounters. When individuals interact with each other repeatedly, they may choose to behave cooperatively, even though this has a negative effect on their short-term interests. Individuals may choose to behave cooperatively because they realize that if they do not behave this way, others won't do either. They also realize that in the long run they are better off in a cooperative world than in a non-cooperative one. Hence, although cooperative behavior harms one's short-term interests, it is likely to be beneficial to one's interests in the long run.

Explaining cooperative behavior in terms of reciprocity assumes that individuals interact with each other repeatedly and that they remember what happened in the past. These assumptions seem reasonable in some contexts but not in others. Because of this, a number of alternative explanations of cooperative behavior have been proposed in the literature. In this paper, we focus on one such explanation. This is the explanation that cooperative behavior is a consequence of the spatial distribution of individuals and the local interaction among them. In the biological literature, this explanation was proposed in a well-known paper by Nowak and May (1992). Many 
biologists have built on this work, which has resulted in a substantial body of literature.

Inspired by the work done in biology, economists have also attempted to explain cooperative behavior in terms of local interaction among spatially distributed individuals. An evolutionary perspective is typically taken, in which individuals are assumed to imitate each other and to randomly experiment with new actions. The first work in the economic literature was done by Bergstrom and Stark (1993) and Eshel, Samuelson, and Shaked (1998). In this work, cooperative behavior was shown to emerge in models in which individuals are organized in a circular structure. A large number of studies have built on this early work. ${ }^{1}$ Studies in the economic literature often focus on rather abstract models. Many studies for example assume that individuals are located in a one-dimensional world. Also, many studies assume a situation similar to a classical prisoners' dilemma, in which individuals can choose from only two actions (i.e., cooperation and defection). For examples of studies that make these assumptions, we refer to Bergstrom and Stark (1993), Eshel et al. (1998), Jun and Sethi (2007), Mengel (2009), and Stark and Behrens (2010).

In this paper, we consider a somewhat less abstract level of modeling. We aim to determine to what extent the findings from earlier studies generalize to models of price competition among spatially distributed firms. In particular, we want to find out whether imitation and experimentation may cause cooperative behavior to emerge in spatial price competition models. Compared with the frequently studied prisoners' dilemma models, the models that we study are of a more complex nature. There is no simple binary decision between cooperative and non-cooperative behavior in our models. Firms can cooperate with each other by jointly increasing their price, and different price levels correspond with different levels of cooperation. Also, interactions in our models may involve more than two individuals. In one of our models, each consumer has four different firms from which he may choose to buy. Hence, firms in this model always have multiple competitors with which they fight for the same market share. Like in the literature mentioned above, we take an evolutionary perspective in our models. We assume firms' behavior to be determined by imitation and experimentation. More specifically, we assume that firms change their price either by imitating successful competitors in their neighborhood or by experimenting with small price increases or decreases.

We study a variety of conditions under which firms may or may not start to cooperate with each other. We consider both a model in which firms are organized in a one-dimensional space and a model in which firms are organized in a twodimensional space. Our two-dimensional model has two variants, which differ from each other in the way in which consumers are located. We also look at the effect of the information firms have about competitors in their neighborhood. In doing so, we distinguish between on the one hand the number of competitors about which firms have information and on the other hand the accuracy of the information firms have. Another effect that we look at is the effect of firms' experimentation probability, that is, the probability with which firms experiment with small price increases or decreases. Due to the complexity of the models that we study, we perform our analyses mainly using computer simulations.

\footnotetext{
${ }^{1}$ See Barr and Tassier (2010), Bilancini and Boncinelli (2009), Chen and Chow (2009), Eshel et al. (2000), Eshel, Sansone, and Shaked (1999), Fosco and Mengel (2011), Jun and Sethi (2007, 2009), Kirchkamp (1999, 2000), Mengel (2009), Noailly, Van den Bergh, and Withagen (2009), Noailly, Withagen, and Van den Bergh (2007), Outkin (2003), Stark and Behrens (2010), Tieman, Houba, and Van der Laan (2000), and Wilhite (2006).
} 
This paper is organized as follows. The models that we study are introduced in Section 2. The analysis of the models is presented in Section 3. The main conclusions of our research are summarized in Section 4.

\section{Models}

We consider two closely related models. We refer to these models as the onedimensional model and the two-dimensional model. The way in which firms and consumers are located is different in each model. Apart from this difference, the models are essentially identical. We discuss the one-dimensional model in Subsection 2.1 and the two-dimensional model in Subsection 2.2.

\subsection{One-dimensional model}

There are $n$ firms, denoted by $1, \ldots, n$. In the one-dimensional model, firms are located equally spaced on a circle (see Figure 1). ${ }^{2}$ The distance, measured over the circumference of the circle, between any two neighboring firms equals one. Consumers are uniformly distributed on the circle. They are modeled as a continuum. The density of consumers equals 1 everywhere on the circle. Firms all produce the same product, they all have an unlimited production capacity, and they all have the same constant marginal cost. Without loss of generality, firms' constant marginal cost is set to zero. The price at which firm $i$ sells one unit of its product is denoted by $p_{i}$. Firms choose their prices simultaneously. A consumer's total cost of buying a unit from a firm equals the price charged by the firm plus transportation cost. Transportation cost equals the distance, measured over the circumference of the circle, between the consumer and the firm. Each consumer needs exactly one unit of the product produced by the firms. A consumer buys this unit from the firm for which the consumer's total cost is lowest. This implies that the circle can be partitioned into $n$ segments in such a way that all consumers located on the ith circle segment buy from firm $i$. Firm i's quantity demanded, denoted by $q_{i}$, then equals the length of the $i$ th circle segment, and firm $i$ 's profit is given by $\pi_{i}=p_{i} q_{i}$.

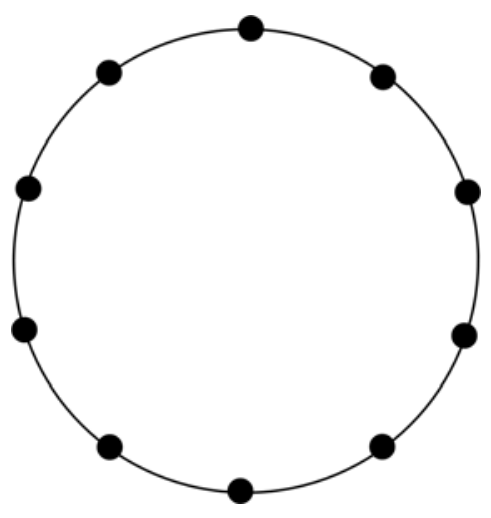

Figure 1. One-dimensional model with $n=10$ firms. A firm is indicated by a black dot. Consumers are located everywhere on the circle.

The model has a symmetric pure-strategy Nash equilibrium in which $p_{1}=\ldots=p_{n}$ $=1$. This can be seen as follows. Suppose that $p_{1}=\ldots=p_{n}=1$. We will show that a firm cannot increase its profit by unilaterally changing its price. Consider an arbitrary

\footnotetext{
${ }^{2}$ Firms are located on a circle rather than on a line in order to avoid boundary effects.
} 
firm $i$, and suppose that this firm changes its price $p_{i}$. The other firms do not change their price. Next, consider a consumer located somewhere in between firm $i$ and firm $j$, where firm $j$ is one of the two neighboring firms of firm $i$. Let the distance between the consumer and firm $i$ be denoted by $d$. The distance between the consumer and firm $j$ is then given by $1-d$. The consumer's total cost of buying from firm $i$ equals $p_{i}+d$, while the consumer's total cost of buying from firm $j$ equals $p_{j}+1-d=2-d$. Hence, the consumer will buy from firm $i$ if $p_{i}+d<2-d$ or, equivalently, if $d<1-p_{i} / 2$. This means that firm i's quantity demanded equals $q_{i}=2\left(1-p_{i} / 2\right)=2-p_{i}$ and that firm $i$ makes a profit of $\pi_{i}=p_{i}\left(2-p_{i}\right)$. Clearly, firm $i$ maximizes its profit by choosing a price of $p_{i}=1$. In other words, if firm $i$ changes its price to a value different from 1 , its profit will decrease. This implies that $p_{1}=\ldots=p_{n}=1$ is a Nash equilibrium. In this equilibrium, each firm makes a profit of 1.

It is straightforward to see that firms find themselves in a situation that is somewhat similar to a prisoners' dilemma. Choosing the Nash equilibrium price can be seen as defection, while choosing a price above the Nash equilibrium level can be seen as cooperation. If a firm cooperates while its neighbors defect, the firm will make a lower profit than in the Nash equilibrium. However, if a firm cooperates and its neighbors do so as well, the firm will make a higher profit than in the Nash equilibrium. The payoff matrix shown in Table 1 illustrates the situation in which firms find themselves. Notice that there is one important difference with a prisoners' dilemma. This is because a firm may also choose a price below the Nash equilibrium level. For this action, there is no analogous action in a prisoners' dilemma.

Table 1. Payoff matrix that illustrates the situation of firms in the one-dimensional model. For the purpose of illustration, price is treated as a discrete variable that can take four different values. The row player represents an arbitrary firm $i$. The column player represents firms $i-1$ and $i+1$, which are the two neighbors of firm $i$. (The two neighbors are assumed to choose the same price.) The payoffs represent the profits of firm $i .^{3}$

\begin{tabular}{l|cccc} 
& $p_{i-1}=p_{i+1}=1.50$ & $p_{i-1}=p_{i+1}=1.25$ & $p_{i-1}=p_{i+1}=1.00$ & $p_{i-1}=p_{i+1}=0.75$ \\
\hline$p_{i}=1.50$ & 1.50 & 1.13 & 0.75 & 0.38 \\
$p_{i}=1.25$ & 1.56 & 1.25 & 0.94 & 0.63 \\
$p_{i}=1.00$ & 1.50 & 1.25 & 1.00 & 0.75 \\
$p_{i}=0.75$ & 1.31 & 1.13 & 0.94 & 0.75
\end{tabular}

In our model, we assume firms to be boundedly rational. Hence, firms need not use Nash equilibrium strategies. We take an evolutionary game theory approach and assume firms' behavior to be determined by imitation and experimentation. The stage game described above is played repeatedly for a large number of rounds. After each round, firms may change their price. Firms change their price by imitating successful neighbors or by experimenting with a small price increase or decrease. Price is modeled as a discrete variable. That is, firms choose their price from a finite set of price levels.

Imitation is modeled as follows. At the end of each round, a firm is randomly selected. The selected firm knows its own price in the most recent round and the

\footnotetext{
${ }^{3}$ In the payoff matrix shown in Table 1, there are three Nash equilibria, namely a strict Nash equilibrium in which each firm charges a price of 1 and two weak Nash equilibria, one in which each firm charges a price of 1.25 and one in which each firm charges a price of 1.5. The weak Nash equilibria are due to the treatment of price as a discrete variable. We will come back to this issue later on in this paper (see Footnote 5).
} 
prices of its $\rho$ closest neighbors, where $\rho$ is an even number that indicates the size of the information neighborhood of a firm. The selected firm also observes its own profit in the most recent round and the profits of its $\rho$ closest neighbors. In the case of its neighbors, however, the firm does not observe their true profits but rather their true profits perturbed by some noise. For each neighbor, the noise is modeled by adding a normally distributed random variable to the neighbor's true profit. The random variable has mean 0 and standard deviation $\sigma$, where we refer to $\sigma$ as the noise level. The selected firm chooses a new price by copying the price that appears to have been most profitable in the most recent round. More specifically, the firm first averages the observed profits of firms that used the same price in the most recent round. The firm then chooses the price associated with the highest observed profit as its new price. (Ties are broken randomly.) An illustration of the imitation mechanism is provided in Figure 2.

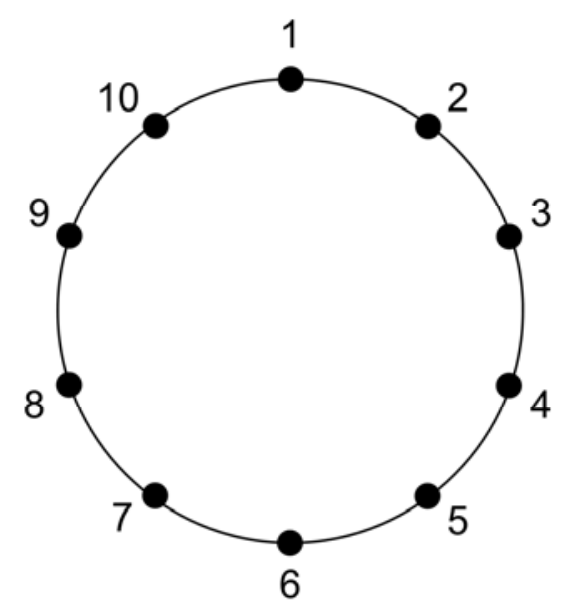

\begin{tabular}{l|cccccccccc} 
& \multicolumn{1}{|c}{ Firm } & \multicolumn{1}{c}{} & \\
& 1 & 2 & 3 & 4 & 5 & 6 & 7 & 8 & 9 & 10 \\
\hline Price & 1.00 & 1.10 & 1.10 & 1.10 & 1.00 & 0.90 & 1.00 & 1.00 & 0.90 & 0.80 \\
Quantity & 0.95 & 0.95 & 1.00 & 0.95 & 1.00 & 1.10 & 0.95 & 0.95 & 1.00 & 1.15 \\
Profit & 0.95 & 1.05 & 1.10 & 1.05 & 1.00 & 0.99 & 0.95 & 0.95 & 0.90 & 0.92 \\
Obs. profit & & & 1.17 & 1.23 & 1.00 & 0.98 & 0.86 & & &
\end{tabular}

Figure 2. Illustration of the imitation mechanism. There are $n=10$ firms. Firms have an information neighborhood of size $\rho=4$, and the noise level equals $\sigma=0.1$. For each firm, the price, the quantity demanded, and the profit are listed in the table. Firm 5 is randomly selected to change its price. Firm 5 does not observe the true profits of its neighbors but rather their true profits perturbed by some noise. The profits observed by firm 5 are listed in the table as well. The average observed profit equals 0.98 for a price of $0.9,(1.00+0.86) / 2=0.93$ for a price of 1.0 , and $(1.17+1.23) / 2$ $=1.20$ for a price of 1.1 . Hence, firm 5 will increase its price from 1.0 to 1.1 .

Experimentation takes place after imitation and is modeled as follows. At the end of each round, each firm independently decides whether to experiment with a new price or not. The probability that a firm chooses to experiment is given by the parameter $\mu$. This probability is typically very small. If a firm chooses to experiment, there is a $50 \%$ probability of a price increase and a $50 \%$ probability of a price decrease. The firm will set its new price to the closest price level above or below its 
current price. Of course, if the firm's current price equals the highest (lowest) price level that can be chosen in the model, no price increase (decrease) is possible.

One-dimensional models have been frequently studied in the literature (Barr \& Tassier, 2010; Bergstrom \& Stark, 1993; Chen \& Chow, 2009; Eshel et al., 1998, 1999, 2000; Jun \& Sethi, 2007; Mengel, 2009; Noailly et al., 2007; Outkin, 2003; Stark \& Behrens, 2010; Wilhite, 2006). ${ }^{4}$ Our model is somewhat similar to the model of Eshel et al. (1998). Eshel et al. study a population of altruists and egoists located on a circle. Like in our model, agents imitate the strategies of successful neighbors. It turns out that, even though being an egoist is a dominant strategy, altruism can still prevail in the long run. Altruism can prevail if altruists are grouped together on the circle, so that they benefit from each other's altruism. An important difference between the model of Eshel et al. and our model is that in our model agents can choose from more than two actions (i.e., firms can choose from more than two price levels). Another difference is that in our model agents do not always have noise-free information about their neighbors' payoffs.

\subsection{Two-dimensional model}

The two-dimensional model is very similar to the one-dimensional model except that firms and consumers are located differently. In the two-dimensional model, we start with a square lattice of $m \times m$ points. There are $n=m^{2}$ firms, which are located on the points of the lattice (see Figure 3). The distance between firms that are direct neighbors equals one. The model has two variants. These variants differ from each other in the way in which consumers are located (see Figure 3). In one variant, referred to as variant A, consumers are located only on line segments between firms that are direct neighbors. In the other variant, referred to as variant $\mathrm{B}$, consumers are located everywhere in the two-dimensional space in between the firms. Consumers are modeled as a continuum in both variants of the model. Also, in both variants, the distribution of consumers is uniform, with a density of one everywhere. All distances in the model are calculated using the city block or Manhattan distance measure rather than using the Euclidean distance measure (see Figure 4). The use of the city block measure is mathematically convenient, but it also is a natural choice if we interpret the model in terms of firms and consumers located in a city with a block design.

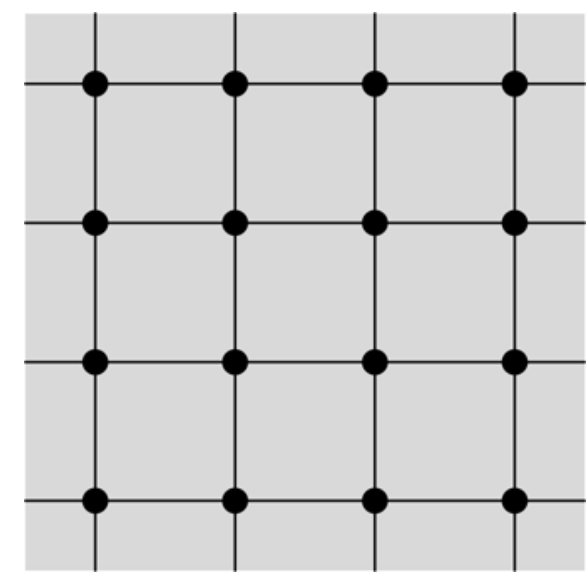

\footnotetext{
${ }^{4} \mathrm{We}$ focus on the theoretical literature. In the experimental literature, a model similar to our onedimensional model is considered by Selten and Apesteguia (2005).
} 
Figure 3. Two-dimensional model with $n=16$ firms. A firm is indicated by a black dot. In variant $A$ of the model, consumers are located only on the black lines. In variant $\mathrm{B}$ of the model, consumers are located everywhere in the gray area.

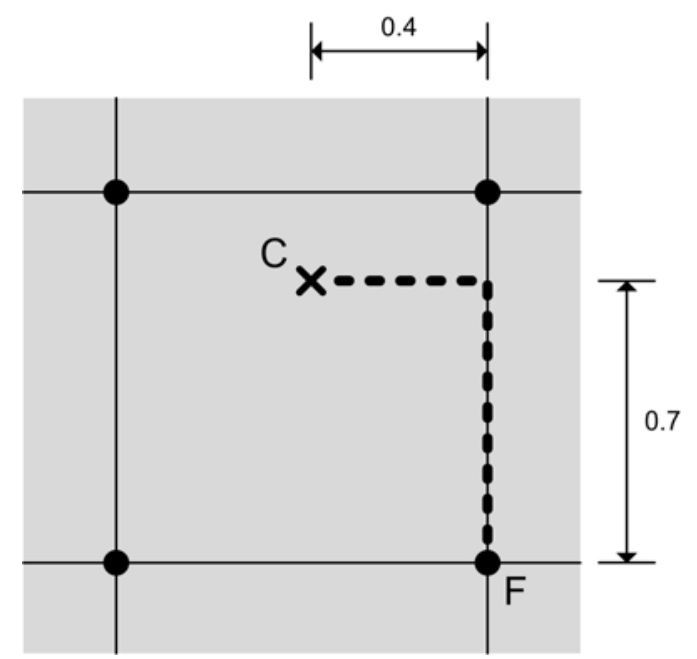

Figure 4. Illustration of the calculation of distances using the city block measure. The distance between firm $\mathrm{F}$ and consumer $\mathrm{C}$ equals $0.7+0.4=1.1$.

In our one-dimensional model discussed in Subsection 2.1, firms and consumers are located on a circle rather than on a line. This simplifies the analysis of the model, because there are no boundary effects that need to be taken into account. In a similar way, we also want to avoid boundary effects in our two-dimensional model. We therefore treat firms at opposite edges of the two-dimensional space as direct neighbors of each other. Hence, each of the leftmost firms has a direct neighbor among the rightmost firms. Similarly, each of the topmost firms has a direct neighbor among the bottommost firms. In this way, there are no boundary effects in the model. That is, each firm finds itself in exactly the same position, with the same number of neighboring firms and an equally-sized consumer market.

Both variants of the model have a symmetric pure-strategy Nash equilibrium. Variant $\mathrm{A}$ has an equilibrium in which $p_{1}=\ldots=p_{n}=1$. This can be shown using an argument analogous to the argument used in the case of the one-dimensional model. Variant $\mathrm{B}$ has an equilibrium in which $p_{1}=\ldots=p_{n}=1 / 2$. This can be seen as follows. Suppose that $p_{1}=\ldots=p_{n}=1 / 2$. We will show that a firm cannot increase its profit by unilaterally changing its price. Consider an arbitrary firm $i$, and suppose that this firm changes its price $p_{i}$. The other firms do not change their price. We distinguish between two cases, namely the case of a price increase and the case of a price decrease. We first analyze the case of a price increase. If firm $i$ increases its price $p_{i}$ to a value above $1 / 2$, its quantity demanded will become $q_{i}=\max \left(3 / 2-p_{i}, 0\right)^{2}$ (see Figure 5(a) for an illustration for $\left.p_{i}=0.7\right)$. Consequently, firm $i$ will make a profit of $\pi_{i}=p_{i}$ $\max \left(3 / 2-p_{i}, 0\right)^{2}$. Clearly, for $p_{i} \geq 1 / 2$, this profit function is monotonically decreasing. Hence, if firm $i$ increases its price to a value above $1 / 2$, its profit will decrease. We now analyze the case of a price decrease. In the case of a price decrease, firm i's quantity demanded is given by $q_{i}=(1 / 2) p_{i}{ }^{2}-(5 / 2) p_{i}+17 / 8$ (see Figure $5(\mathrm{~b})$ for an illustration for $\left.p_{i}=0.1\right)$. This results in a profit function of $\pi_{i}=(1 / 2) p_{i}{ }^{3}-$ $(5 / 2) p_{i}^{2}+(17 / 8) p_{i}$. For $p_{i} \leq 1 / 2$, this function is monotonically increasing. This implies that a decrease of firm $i$ 's price to a value below $1 / 2$ will lead to a decrease of firm $i$ 's 
profit. Hence, both a price increase and a price decrease will lead to a decrease in profit. This shows that $p_{1}=\ldots=p_{n}=1 / 2$ is a Nash equilibrium of variant $\mathrm{B}$ of the model.

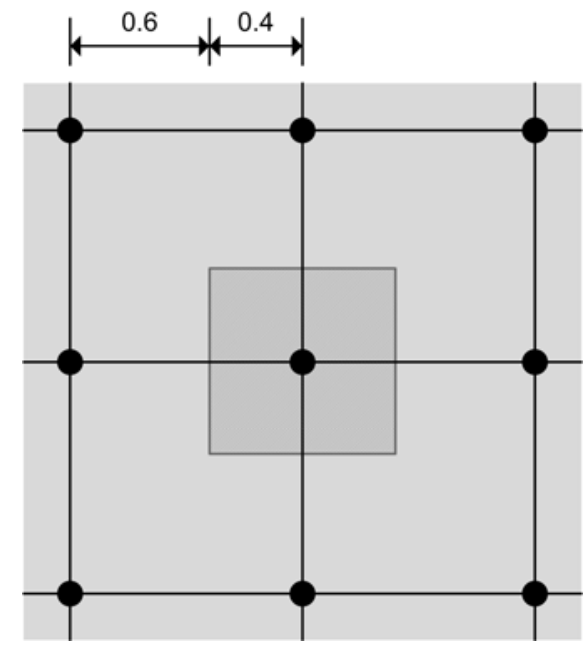

(a)

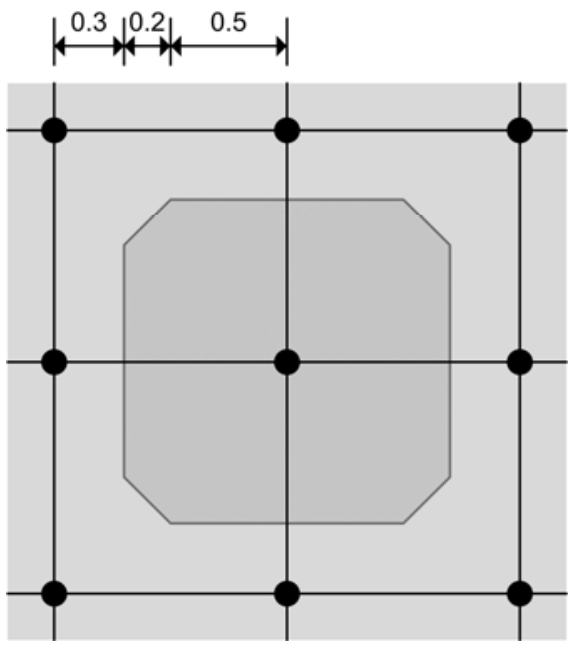

(b)

Figure 5. Illustration of the calculation of a firm's quantity demanded. The firm in the center of panel (a) charges a price of 0.7 . The firm in the center of panel (b) charges a price of 0.1 . The surrounding firms all charge a price of 0.5 . In both panels, the shaded area marks the consumers that buy from the firm in the center. In panel (a), the quantity demanded of the firm in the center equals $0.8 \times 0.8=0.64$. In panel (b), the quantity demanded of the firm in the center equals $1.4 \times 1.4-4 \times 0.5 \times 0.2 \times 0.2=$ 1.88 .

Both in variant $\mathrm{A}$ and in variant $\mathrm{B}$ of the model, firms find themselves in a situation that resembles a prisoners' dilemma. If a single firm unilaterally deviates from the Nash equilibrium by increasing its price, the firm will make a lower profit than in the equilibrium. However, if all firms jointly deviate from the Nash equilibrium by increasing their price, they will all make a higher profit than in the equilibrium.

Like in our one-dimensional model, we assume firms to be boundedly rational in our two-dimensional model. Firms' behavior is assumed to be determined by imitation and experimentation in the same way as in the one-dimensional model. In the one-dimensional model, imitation takes place by looking at the prices and profits of $\rho+1$ firms, namely a randomly selected firm and its $\rho$ closest neighbors. In the two-dimensional model, we focus on two scenarios for the way in which firms imitate each other. In the first scenario, imitation takes place based on the prices and profits of five firms, namely a randomly selected firm and its direct neighbors in horizontal and vertical direction. In this scenario, firms have an information neighborhood of size $\rho=4$. In the second scenario, imitation takes place based on the prices and profits of nine firms. In this scenario, the selected firm's direct neighbors in diagonal direction are included as well. The second scenario results in an information neighborhood of size $\rho=8$. 
A number of two-dimensional models have been studied in the economic literature (Barr \& Tassier, 2010; Kirchkamp, 1999, 2000; Noailly et al., 2009; Outkin, 2003; Tieman et al., 2000; Wilhite, 2006). The models of Kirchkamp (2000) and Tieman et al. (2000) are the ones that are most closely related to our model. Kirchkamp studies a two-dimensional model in which agents play prisoners' dilemma games with their neighbors. He shows that under certain conditions cooperation can prevail in the long run. Important differences between Kirchkamp's model and our model are that in our model agents can choose from more than two actions and that in our model agents do not always have noise free information about their neighbors' payoffs. Tieman et al. study a local interaction model in which agents play generalized prisoners' dilemma games, that is, prisoners' dilemma games in which there can be more than two actions. They find that with a high probability a moderate level of cooperation emerges in their model. An essential difference between the model of Tieman et al. and our model is that in the model of Tieman et al. agents do not imitate each others' strategies. Instead, agents increase or decrease their cooperativeness depending on whether their average payoff is higher or lower than the average payoff of their neighbors.

\section{Analysis}

We are interested in the long-run behavior of firms in our one-dimensional and two-dimensional models. In particular, we want to find out whether in the long run firms behave cooperatively by charging prices above the Nash equilibrium level. Because our models do not seem analytically tractable, we use computer simulations to perform our analysis. In Subsection 3.1, the setup of the simulations is discussed. The results obtained using the simulations are presented in Subsections 3.2 (onedimensional model) and 3.4 (two-dimensional model). Some intuitive insight into the one-dimensional model is provided in Subsection 3.3.

\subsection{Simulation setup}

In our simulations, there are $n=400$ firms. This means that in the twodimensional model firms are located on the points of a $20 \times 20$ square lattice. Firms choose their price from a set of 21 price levels. These price levels are uniformly distributed between $0.5 p_{\mathrm{N}}$ and $1.5 p_{\mathrm{N}}$, where $p_{\mathrm{N}}$ denotes the Nash equilibrium price. Hence, in the one-dimensional model and in variant A of the two-dimensional model, the price levels that can be chosen are $0.50,0.55, \ldots, 1.50$. In variant $B$ of the twodimensional model, the price levels that can be chosen are $0.250,0.275, \ldots, 0.750 .^{5} \mathrm{In}$ the case of the one-dimensional model, simulations are run for six different values of the information neighborhood size $\rho$, namely $2,4,6,8,10$, and 20 . In the case of the two-dimensional model, simulations are run for an information neighborhood of size $\rho=4$ and for an information neighborhood of size $\rho=8$. Furthermore, both in the case of the one-dimensional model and in the case of the two-dimensional model, simulations are run for four different noise levels $\sigma$, namely $0,0.1 p_{\mathrm{N}}, 0.2 p_{\mathrm{N}}$, and

\footnotetext{
${ }^{5}$ Modeling price as a discrete rather than a continuous variable may introduce additional Nash equilibria. This turns out to be the case in the one-dimensional model and in variant A of the twodimensional model. In addition to a strict Nash equilibrium in which each firm charges a price of 1.00 , there are two weak Nash equilibria in these models, one in which each firm charges a price of 0.95 and one in which each firm charges a price of 1.05. There turn out to be no additional Nash equilibria in variant B of the two-dimensional model. In the rest of this paper, when we refer to a Nash equilibrium of a model, we always mean a strict Nash equilibrium.
} 
$0.5 p_{\mathrm{N}}$, and for four different experimentation probabilities $\mu$, namely $0,0.00001$, 0.0001 , and 0.001 .

At the beginning of a simulation run, each firm's price is initialized by randomly drawing a price from a uniform distribution over all price levels. A simulation run lasts for one million rounds. One million rounds turns out to be sufficient for studying firms' long-run behavior. We performed some tests which indicate that after one million rounds the results of our simulations are insensitive to the way in which firms' prices have been initialized. The tests that we performed also indicate that a larger number of rounds would yield essentially the same simulation results.

The source code of the simulations is available online at www.ludowaltman.nl/price competition/. The source code runs in MATLAB and has been written partly in the MATLAB language and partly in the $C$ language.

\subsection{Simulation results for the one-dimensional model}

The results of the simulations for the one-dimensional model are reported in Tables 2 and 3. For each combination of an information neighborhood size $\rho$, a noise level $\sigma$, and an experimentation probability $\mu, 500$ simulation runs were performed. For each simulation run, we calculated the mean price of the firms at the end of the last round (i.e., at the end of the one millionth round). In Tables 2 and 3, this mean price is averaged over the 500 simulations runs that were performed. Standard deviations over the 500 simulation runs (not reported in the tables) are always less than 0.05 . The relatively small standard deviations indicate that there is little variation between simulation runs.

Table 2. Simulation results for the one-dimensional model for $\rho=2$ and for different values of $\sigma$ and $\mu$. The table shows the mean price at the end of the simulation runs.

\begin{tabular}{l|cccc} 
& $\sigma=0.0$ & $\sigma=0.1$ & $\sigma=0.2$ & $\sigma=0.5$ \\
\hline$\mu=0$ & 1.28 & 1.33 & 1.31 & 1.31 \\
$\mu=0.00001$ & 1.27 & 1.25 & 1.27 & 1.28 \\
$\mu=0.0001$ & 1.25 & 1.20 & 1.22 & 1.23 \\
$\mu=0.001$ & 1.14 & 1.11 & 1.13 & 1.15
\end{tabular}

Table 3. Simulation results for the one-dimensional model for different values of $\rho, \sigma$, and $\mu$. The table shows the mean price at the end of the simulation runs.

\begin{tabular}{l|cccc} 
& $\sigma=0.0 ; \mu=0$ & $\sigma=0.0 ; \mu=0.0001$ & $\sigma=0.2 ; \mu=0$ & $\sigma=0.2 ; \mu=0.0001$ \\
\hline$\rho=2$ & 1.28 & 1.25 & 1.31 & 1.22 \\
$\rho=4$ & 0.90 & 0.90 & 0.93 & 0.95 \\
$\rho=6$ & 0.90 & 0.90 & 0.95 & 0.95 \\
$\rho=8$ & 0.90 & 0.93 & 0.95 & 0.96 \\
$\rho=10$ & 0.93 & 0.95 & 0.96 & 0.96 \\
$\rho=20$ & 0.98 & 1.00 & 0.98 & 0.98
\end{tabular}

In Table 2, results are reported of simulations in which the information neighborhood has a size of $\rho=2$. In these simulations, firms can imitate only their direct neighbors. As can be seen in the table, the simulations yield prices that are substantially above the Nash equilibrium level of 1 . The prices are not very sensitive to the noise level $\sigma$. They are somewhat more sensitive to the experimentation probability $\mu$. A higher experimentation probability clearly leads to a lower price. The results in Table 2 are in line with the findings of earlier studies in which somewhat similar models were analyzed (e.g., Eshel et al., 1998). 
We now turn to the effect of the information neighborhood size $\rho$. Simulation results for different values of the size of the information neighborhood are reported in Table 3. If the size of the information neighborhood is larger than 2, firms can imitate not only their direct neighbors but also some of their more distant neighbors. The results in Table 3 are quite remarkable. It turns out that prices are no longer above the Nash equilibrium level if the size of the information neighborhood is larger than 2. On the contrary, if the size of the information neighborhood is not too large, prices turn out to be below the Nash equilibrium level. This is especially the case if there is no noise and no experimentation (i.e., $\sigma=0$ and $\mu=0$ ). In earlier studies (Hoffmann, 1999; Ifti, Killingback, \& Doebeli, 2004; Mengel, 2009; Stark \& Behrens, 2010), it was found that cooperative behavior (i.e., prices above the equilibrium level in our context) tends to be more difficult to sustain if the size of the information neighborhood is increased. This is consistent with our findings, but our findings go one step further. If the size of the information neighborhood is increased, firms not only stop behaving cooperatively but they in fact start behaving in exactly the opposite way, that is, they decrease their prices to values below the equilibrium level. Hence, our results show that in some cases the combination of local interaction and imitation of neighboring individuals has a negative rather than a positive effect on the degree to which individuals cooperate with each other.

\subsection{Further analysis of the one-dimensional model}

Why does our one-dimensional model yield completely opposite simulation results for an information neighborhood of size $\rho=2$ on the one hand and for an information neighborhood of size $\rho \in\{4,6,8,10\}$ on the other hand? To provide some intuitive insight, we first focus on the case of an information neighborhood of size 2 and we then consider the case of an information neighborhood of size 4. To simplify the analysis, we assume that in both cases firms can choose from only two price levels. We also assume that there is no noise and no experimentation (i.e., $\sigma=0$ and $\mu=0$ ). In other words, the only way in which a firm can change its price is by means of imitation, and if a firm imitates, it does so based on noise-free information about the profits of its neighbors.

In the case of an information neighborhood of size 2, we assume that firms charge a price of either 1.0 (i.e., the Nash equilibrium price) or $1.1{ }^{6}$ We refer to these prices as, respectively, the low price and the high price, and we refer to firms charging the low price as low-price firms and to firms charging the high price as high-price firms. Suppose that we have a cluster of low-price firms and a cluster of high-price firms. By a cluster of low-price (high-price) firms, we mean a number of low-price (high-price) firms that are direct neighbors of each other. Suppose further that the cluster of lowprice firms and the cluster of high-price firms are located next to each other in the one-dimensional space of our model. This is illustrated in Figure 6(a). The figure also shows the profit made by each firm. Based on Figure 6(a), let us look what will happen. A low-price firm that is surrounded by two other low-price firms cannot change its price. The same holds for a high-price firm that is surrounded by two other high-price firms. We therefore focus on firms 5 and 6 in Figure 6(a). Firm 6 will not change its price. This is because, based on the information available to this firm, the average profit resulting from the high price (i.e., $\pi_{6} / 2+\pi_{7} / 2 \approx 1.073$ ) exceeds the average profit resulting from the low price (i.e., $\pi_{5}=1.050$ ). Hence, firm 6 will stick

\footnotetext{
${ }^{6}$ The choice of these two prices is fairly arbitrary. However, our analysis is valid for many other prices as well.
} 
to the high price. Unlike firm 6 , firm 5 will change its price. Firm 5 is currently a lowprice firm, but based on the information available to the firm, the high price appears to be more profitable than the low price (since $\pi_{6}=1.045>\pi_{4} / 2+\pi_{5} / 2=1.025$ ). As a consequence, firm 5 will change to the high price. This will lead to a new situation, which is illustrated in Figure 6(b). Looking at Figure 6(b), it is clear that the next step will be firm 4 changing from the low price to the high price. Hence, the general pattern is that the cluster of high-price firms is growing more and more while the cluster of low-price firms is shrinking. This is the basic intuition underlying our simulation results for an information neighborhood of size $2 .^{7}$
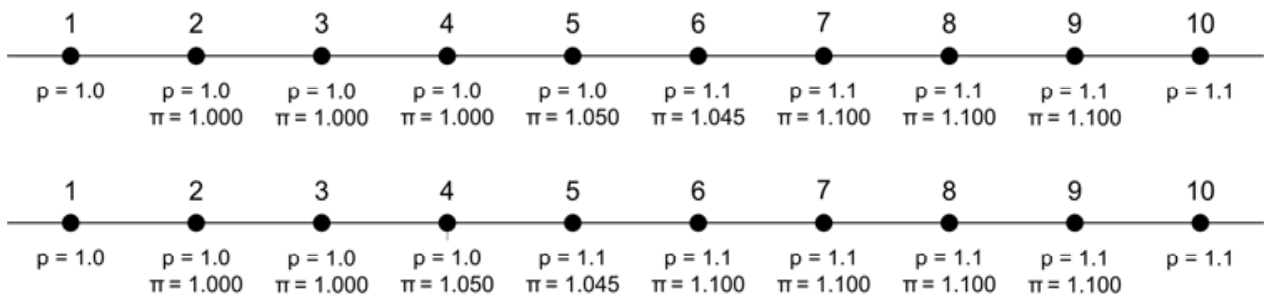

Figure 6. Illustration of the effect of imitation in the one-dimensional model with an information neighborhood of size $\rho=2$.

We now consider the case of an information neighborhood of size $4 .{ }^{8}$ In this case, we assume that firms charge either a low price of 0.9 or a high price of 1.0 (i.e., the Nash equilibrium price). Notice that these prices are different from the prices used in the above analysis for an information neighborhood of size 2 . This is because we now want to explain why firms charge prices below the Nash equilibrium level, while in the analysis presented above we wanted to explain why firms charge prices above the Nash equilibrium level. We again start from a situation with a cluster of low-price firms and a cluster of high-price firms. This situation is illustrated in Figure 7(a). Based on Figure 7(a), it can be seen that there are two firms for which a price change is possible, namely firm 4 and firm 5 . In both cases, there would be a change from the low price to the high price. Firm 4 and firm 5 cannot both change their price at the same time. Instead, one of the two firms will be randomly selected to change its price. If firm 5 is selected, the effect will be that the cluster of high-price firms grows while the cluster of low-price firms shrinks. This is similar to what happens in the case of an information neighborhood of size 2. If firm 4 is selected, the effect will be quite different. We will then end up in the situation illustrated in Figure 7(b). As can be seen in the figure, there will no longer be a perfect separation of low-price and highprice firms. In this new situation, there turn out to be three firms for which a price

\footnotetext{
${ }^{7}$ The full story is more complicated. In particular, it can be shown that low-price firms will not disappear altogether. Suppose we have a cluster of low-price firms surrounded on both sides by a cluster of high-price firms. As explained above, the cluster of low-price firms will shrink more and more. However, when there are just two low-price firms left, the cluster of low-price firms will not shrink any further. Hence, in the end there will be mostly high-price firms, but in between these firms there will also be some small islands of low-price firms. We refer to Eshel et al. (1998) for an extensive discussion of this kind of dynamics.

${ }^{8}$ We refer to Mengel (2009) for a somewhat similar analysis. One of the differences between our analysis and the analysis of Mengel is that we do not consider the effect of experimentation while Mengel focuses on the limit case in which the probability of experimentation (referred to as trembling by Mengel) approaches zero.
} 
change is possible, namely firm 4 , firm 6 , and firm 7. In all three cases, the price change would be a movement from the high price to the low price. At this point, a comprehensive analysis of the various possibilities becomes cumbersome. Let us therefore focus on the most interesting possibility. This is the possibility of a price change by firm 7. If firm 7 changes its price, the cluster of high-price firms will shrink, as is illustrated in Figure 7(c). A next step could then be that firm 8 or firm 9 also changes its price, which would mean that the cluster of high-price firms will shrink even further. Going back to the initial situation illustrated in Figure 7(a), it is now clear that there are two counteracting forces at work. On the one hand the cluster of high-price firms may grow, while on the other hand this cluster may shrink. In the situation illustrated in Figure 7(a), the cluster of high-price firms will grow if firm 5 changes its price. On the other hand, if firm 4 changes its price, this may cause the cluster of high-price firms to shrink. It is not immediately clear which of these two counteracting forces is stronger. However, based on the simulation results reported in Table 3, it can be concluded that the force working against the high-price firms must be the stronger one.

(a)

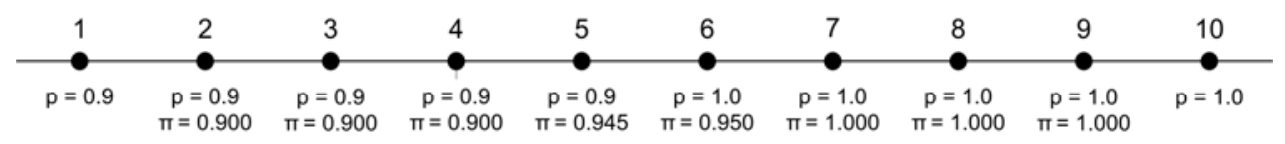

(b)

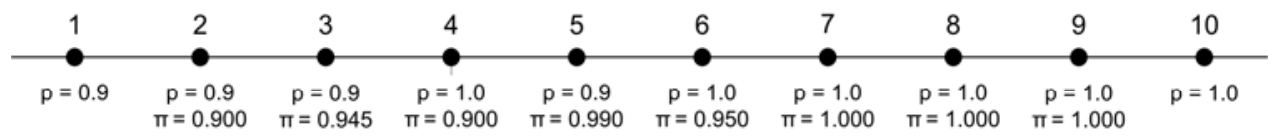

(c)

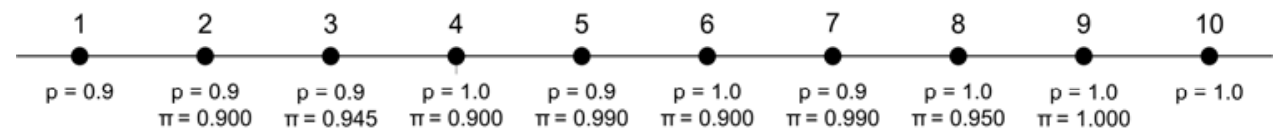

Figure 7. Illustration of the effect of imitation in the one-dimensional model with an information neighborhood of size $\rho=4$.

We have now looked at our one-dimensional model both in the case of an information neighborhood of size 2 and in the case of an information neighborhood of size 4. What is the essential difference between these two cases? In both cases, a cluster of high-price firms may take over a neighboring low-price firm. However, the difference is that in the case of an information neighborhood of size 2 the high-price firms will always remain organized in a single cluster (see Figure 6) while in the case of an information neighborhood of size 4 the high-price firms may become separated from each other (see Figure 7). When high-price firms are separated from each other, they become vulnerable. This is because an isolated high-price firm makes a relatively low profit while an isolated low-price firm makes a relatively high profit. The result may therefore be that low-price firms start to take over high-price firms. This is the basic mechanism that explains why in our one-dimensional model prices are lower in the case of an information neighborhood of size 4 than in the case of an information neighborhood of size 2 .

\subsection{Simulation results for the two-dimensional model}

The results of the simulations for the two-dimensional model are reported in Tables 4 to 7 . Tables 4 and 5 relate to variant $\mathrm{A}$ of the model. This is the variant in 
which consumers are located on line segments between neighboring firms. Tables 6 and 7 relate to variant $\mathrm{B}$ of the model. In this variant, consumers are located everywhere in the two-dimensional space in between the firms. The results in Tables 4 to 7 were obtained in the same way as the results in Tables 2 and 3. Hence, the results are averages over 500 simulation runs. Standard deviations over the 500 simulation runs (not reported in the tables) are always less than 0.03 , indicating that there is little variation between simulation runs.

Table 4. Simulation results for variant A of the two-dimensional model for $\rho=4$ and for different values of $\sigma$ and $\mu$. The table shows the mean price at the end of the simulation runs.

\begin{tabular}{l|cccc} 
& $\sigma=0.0$ & $\sigma=0.1$ & $\sigma=0.2$ & $\sigma=0.5$ \\
\hline$\mu=0$ & 1.15 & 1.05 & 1.06 & 1.06 \\
$\mu=0.00001$ & 1.17 & 1.07 & 1.06 & 1.07 \\
$\mu=0.0001$ & 1.18 & 1.10 & 1.08 & 1.08 \\
$\mu=0.001$ & 1.16 & 1.12 & 1.11 & 1.11
\end{tabular}

Table 5. Simulation results for variant A of the two-dimensional model for $\rho=8$ and for different values of $\sigma$ and $\mu$. The table shows the mean price at the end of the simulation runs.

\begin{tabular}{l|cccc} 
& $\sigma=0.0$ & $\sigma=0.1$ & $\sigma=0.2$ & $\sigma=0.5$ \\
\hline$\mu=0$ & 1.04 & 1.01 & 1.00 & 0.99 \\
$\mu=0.00001$ & 1.04 & 1.00 & 1.00 & 1.00 \\
$\mu=0.0001$ & 1.05 & 1.01 & 1.01 & 1.01 \\
$\mu=0.001$ & 1.07 & 1.05 & 1.04 & 1.03
\end{tabular}

Table 6. Simulation results for variant B of the two-dimensional model for $\rho=4$ and for different values of $\sigma$ and $\mu$. The table shows the mean price at the end of the simulation runs.

\begin{tabular}{l|cccc} 
& $\sigma=0.00$ & $\sigma=0.05$ & $\sigma=0.10$ & $\sigma=0.25$ \\
\hline$\mu=0$ & 0.58 & 0.53 & 0.53 & 0.54 \\
$\mu=0.00001$ & 0.59 & 0.54 & 0.54 & 0.54 \\
$\mu=0.0001$ & 0.59 & 0.55 & 0.55 & 0.55 \\
$\mu=0.001$ & 0.59 & 0.56 & 0.56 & 0.57
\end{tabular}

Table 7. Simulation results for variant B of the two-dimensional model for $\rho=8$ and for different values of $\sigma$ and $\mu$. The table shows the mean price at the end of the simulation runs.

\begin{tabular}{l|cccc} 
& $\sigma=0.00$ & $\sigma=0.05$ & $\sigma=0.10$ & $\sigma=0.25$ \\
\hline$\mu=0$ & 0.52 & 0.50 & 0.51 & 0.51 \\
$\mu=0.00001$ & 0.52 & 0.51 & 0.51 & 0.51 \\
$\mu=0.0001$ & 0.53 & 0.51 & 0.51 & 0.52 \\
$\mu=0.001$ & 0.54 & 0.53 & 0.53 & 0.53
\end{tabular}

We first focus on variant $\mathrm{A}$ of the two-dimensional model. As can be seen in Tables 4 and 5, prices tend to be relatively close to the Nash equilibrium level of 1 . They do not exceed the equilibrium level by more than $18 \%$. This is much less than in the one-dimensional model, in which prices exceed the equilibrium level by at most $33 \%$ (see Table 2). Also, in variant A of the two-dimensional model, prices do not fall 
below the equilibrium level (except for $\sigma=0.5$ and $\mu=0$, where the price is marginally below the equilibrium level). This is another difference with the onedimensional model. In the one-dimensional model, prices can be up to $10 \%$ below the equilibrium level (see Table 3). Comparing Tables 4 and 5, it can be seen that increasing the size of the information neighborhood from $\rho=4$ to $\rho=8$ leads to substantially lower prices. This is similar to what was observed for the onedimensional model, and it is also somewhat similar to earlier findings reported in the literature (Ifti, Killingback, \& Doebeli, 2004). The effect of the noise level $\sigma$ and the experimentation probability $\mu$ is different than in the one-dimensional model. The noise level turns out to have a negative effect on prices, while the experimentation probability turns out to have a positive effect. Notice, however, that especially the effect of the experimentation probability is not very strong.

We now consider variant $B$ of the two-dimensional model. As discussed in Subsection 2.2, variant B has a Nash equilibrium price of 0.5 , which is only half of the Nash equilibrium price of variant A. This explains why the prices in Tables 6 and 7 are much lower than the prices in Tables 4 and 5. When looking at prices relative to the equilibrium price, it can be seen that the results in Tables 6 and 7 are in fact very similar to the results in Tables 4 and 5. The effects of the information neighborhood size $\rho$, the noise level $\sigma$, and the experimentation probability $\mu$ are also very similar. Hence, it turns out that the way in which firms behave is very similar in the two variants of the two-dimensional model.

\section{Conclusions}

We have studied evolutionary models of price competition among spatially distributed firms. In our models, firms are organized either in a one-dimensional space or in a two-dimensional space. Firms' behavior is determined by imitation and experimentation. Imitation means that firms copy the price of one or more successful competitors in their neighborhood. Experimentation means that firms randomly increase or decrease their price by a small amount.

In earlier studies (e.g., Bergstrom \& Stark, 1993; Eshel et al., 1998; Nowak \& May, 1992), often in the context of prisoners' dilemma games, it was found that spatially distributed individuals that interact locally and that imitate successful neighbors tend to behave cooperatively in many cases. In this paper, our aim has been to investigate whether a similar tendency towards cooperative behavior can be found in the context of price competition among spatially distributed firms. In this context, cooperative behavior would mean that firms have prices and profits above the ordinary equilibrium level.

We have performed our analyses mainly using computer simulations. The results of the simulations provide a mixed picture. The emergence of cooperative behavior turns out to depend strongly on the amount of information available to firms. In the one-dimensional model, firms behave cooperatively only if the information they have about the prices and profits of other firms is restricted to their two direct neighbors. In the two-dimensional model, firms behave more cooperatively if they have information about four neighbors than if they have information about eight neighbors. Hence, the general pattern seems to be that having too much information may hurt cooperation (for similar results, see Hoffmann, 1999; Ifti, Killingback, \& Doebeli, 2004; Mengel, 2009; Stark \& Behrens, 2010). We have shown that in the one-dimensional model this is because having too much information may cause cooperative firms to become separated from each other, which weakens their position relative to non-cooperative firms. The two-dimensional model is more difficult to analyze, but the mechanism at 
work in this model may well be similar. A remarkable finding is that in the onedimensional model having too much information may even lead to prices and profits below the ordinary equilibrium level. This shows that the combination of local interaction and imitation of neighboring individuals can have both a positive and a negative effect on the degree to which individuals cooperate with each other. To the best of our knowledge, negative effects have not been reported before in the literature. We have also investigated a number of other factors that may affect the degree of cooperative behavior among firms. One of these factors is the accuracy of the information firms have about the profits of their neighbors. Another factor is the probability with which firms experiment with small price increases or decreases. The effect of these two factors turns out to be relatively small. In the case of the twodimensional model, we have also looked at the effect of the way in which consumers are located in the two-dimensional space. There turn out to be no substantial differences between the two variants that we have considered.

\section{Acknowledgement}

We would like to thank Cornelis van Bochove for his comments on an earlier draft of this paper.

\section{References}

Barr, J., \& Tassier, T. (2010). Endogenous neighborhood selection and the attainment of cooperation in a spatial prisoner's dilemma game. Computational Economics, 35(3), 211-234.

Bergstrom, T.C., \& Stark, O. (1993). How altruism can prevail in an evolutionary environment. American Economic Review, 83(2), 149-155.

Bilancini, E., \& Boncinelli, L. (2009). The co-evolution of cooperation and defection under local interaction and endogenous network formation. Journal of Economic Behavior and Organization, 70(1-2), 186-195.

Chen, H.-C., \& Chow, Y. (2009). Evolutionary prisoner's dilemma games with onedimensional local interaction and imitation. Advances in Applied Probability, 41(1), 154-176.

Eshel, I., Herreiner, D.K., Samuelson, L., Sansone, E., \& Shaked, A. (2000). Cooperation, mimesis, and local interaction. Sociological Methods and Research, 28(3), 341-364.

Eshel, I., Samuelson, L., \& Shaked, A. (1998). Altruists, egoists, and hooligans in a local interaction model. American Economic Review, 88(1), 157-179.

Eshel, I., Sansone, E., \& Shaked, A. (1999). The emergence of kinship behavior in structured populations of unrelated individuals. International Journal of Game Theory, 28(4), 447-463.

Fosco, C., \& Mengel, F. (2011). Cooperation through imitation and exclusion in networks. Journal of Economic Dynamics and Control, 35(5), 641-658.

Hoffmann, R. (1999). The independent localisations of interaction and learning in the repeated prisoner's dilemma. Theory and Decision, 47(1), 57-72.

Ifti, M., Killingback, T., \& Doebeli, M. (2004). Effects of neighbourhood size and connectivity on the spatial continuous prisoner's dilemma. Journal of Theoretical Biology, 231(1), 97-106.

Jun, T., \& Sethi, R. (2007). Neighborhood structure and the evolution of cooperation. Journal of Evolutionary Economics, 17(5), 623-646.

Jun, T., \& Sethi, R. (2009). Reciprocity in evolving social networks. Journal of Evolutionary Economics, 19(3), 379-396. 
Kirchkamp, O. (1999). Simultaneous evolution of learning rules and strategies. Journal of Economic Behavior and Organization, 40(3), 295-312.

Kirchkamp, O. (2000). Spatial evolution of automata in the prisoners' dilemma. Journal of Economic Behavior and Organization, 43(2), 239-262.

Mengel, F. (2009). Conformism and cooperation in a local interaction model. Journal of Evolutionary Economics, 19(3), 397-415.

Noailly, J., Van den Bergh, J.C.J.M., \& Withagen, C.A. (2009). Local and global interactions in an evolutionary resource game. Computational Economics, 33(2), $155-173$.

Noailly, J., Withagen, C.A., \& Van den Bergh, J.C.J.M. (2007). Spatial evolution of social norms in a common-pool resource game. Environmental and Resource Economics, 36(1), 113-141.

Nowak, M.A., \& May, R.M. (1992). Evolutionary games and spatial chaos. Nature, 359, 826-829.

Outkin, A.V. (2003). Cooperation and local interactions in the prisoners' dilemma game. Journal of Economic Behavior and Organization, 52(4), 481-503.

Selten, R., \& Apesteguia, J. (2005). Experimentally observed imitation and cooperation in price competition on the circle. Games and Economic Behavior, 51(1), 171-192.

Stark, O., \& Behrens, D.A. (2010). An evolutionary edge of knowing less (or: On the 'curse' of global information). Journal of Evolutionary Economics, 20(1), 77-94.

Tieman, A.F., Houba, H., \& Van der Laan, G. (2000). On the level of cooperative behavior in a local-interaction model. Journal of Economics, 71(1), 1-30.

Wilhite, A. (2006). Economic activity on fixed networks. In L. Tesfatsion \& K.L. Judd (Eds.), Handbook of computational economics, Volume 2 (pp. 1014-1045). Elsevier. 